population census of the National Institute of Statistics, with an estimated prevalence of RA of $0,5 \%$ (women: $0,8 \%$, men:0,2\%). Crude and adjusted rates of the solid tumors were calculated. The trend was analyzed by Generalized Linear Model (GLM).

Results: 338.343 RA hospital admissions were detected in the study period, being $18.401(5,4 \%)$ due to solid tumors. The main clinical-demographic characteristics are shown in the next table.

\begin{tabular}{lllll}
\hline & Total & Women & Men & $P$ value \\
\hline N (\%) & $18.401(5,4)$ & $8689(3,8)$ & $9712(8,6)$ & $P<0,001$ \\
Age, mean (SD) & $69,98(11,21)$ & $68,62(12,3)$ & $71,20(9,99)$ & $P<0,001$ \\
In-hospital exitus n (\%) & $2455(13,34)$ & $1035(11,9)$ & $1420(14,62)$ & $P<0,001$ \\
Charlson Index, mean (SD) & $5,72(2,95)$ & $5,63(2,95)$ & $5,80(2,95)$ & $P<0,001$ \\
Stay, mean (SD) & $10,94(11,6)$ & $10,76(11,6)$ & $11,1(11,77)$ & $P=0,051$ \\
\hline
\end{tabular}

The solid tumor adjusted rate during the study period was $647,53 / 10^{5}$ inhabitants/ yr $\left(366,97 / 10^{5}\right.$ in women and $1792,99 / 10^{5}$ in men; relative risk men:women $\left.=4,8\right)$. This rate increased from $305,65 / 10^{5}$ in 1999 to $993,19 / 10^{5}$ inhabitants/yr in 2015 (from $814,06 / 10^{5}$ in 1999 to $2535,5 / 10^{5}$ in 2015 in men; from $181,68 / 10^{5}$ in 1999 to $607,71 / 10^{5}$ in 2015 in women). The annual age-adjusted rate increased significantly: $7,37 \%(6,52 \%$ in men and $8,02 \%$ in women; $p \leq 0,001)$. figure 1

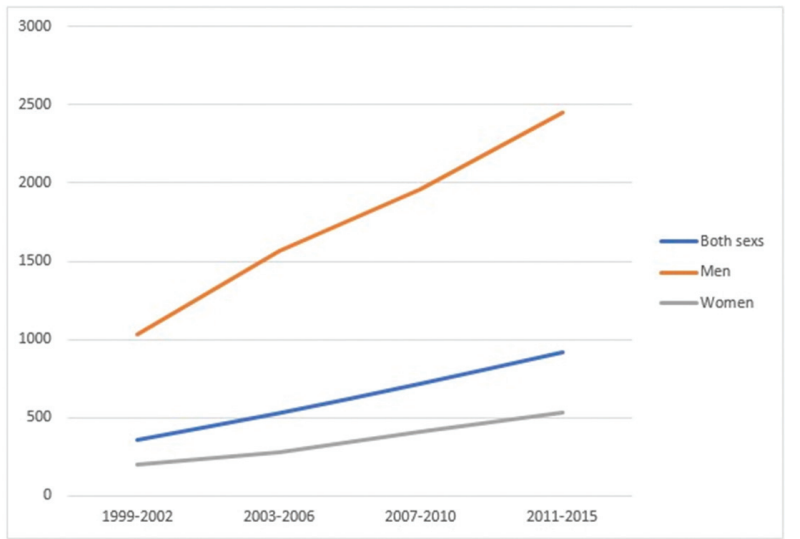

Conclusions: There was an increasing incidence of hospital admissions due to solid tumors in RA in Spain during the period 1999-2015. An annual rate increase of $7.37 \%$, is estimated.

Disclosure of Interest: None declared

DOI: 10.1136/annrheumdis-2018-eular.3346

\section{SAT0115 TRENDS IN THE ACTIVITY OF RHEUMATOID ARTHRITIS AS THE CONSEQUENCE OF TREAT-TO-TARGET STRATEGY: EIGHT-YEAR DATA FROM 2009 TO 2016}

W. Xie ${ }^{1}$, J. Li ${ }^{1}$, X. Zhang ${ }^{1}$, G. Li ${ }^{1}$, Y. Hao ${ }^{1}$, J. Zhao ${ }^{1}$, L. Wang ${ }^{1}$, X. Sun ${ }^{1}$, Y. Fan ${ }^{1}$, Z. Zhang ${ }^{1}$. ${ }^{1}$ Department of Rheumatology and Clinical Immunology, Peking University First Hospital, Beijing, China

Background: In past decades, treatment of rheumatoid arthritis (RA) has advanced greatly, driven largely by the advent of new medications and treat-totarget (T2T) strategy, but the secular trends in the activity and remission of RA over past years and the efficacy of T2T strategy are not fully validated in large population in real life practice.

Objectives: To investigate the trends in the activity of RA over past 8 years and evaluate the value of T2T strategy in daily practice.

Methods: All the medical records of RA patients from 2009 to 2016 were retrospectively reviewed. Disease activity scores at obtained visits were measured by DAS28-CRP, DAS28-ESR, SDAI and CDAI. To display trends over years, both mean and time-adjusted methods were applied in calculation of annual disease activity and remission rate. Disease activity and remission rate were also compared before and after the year of 2011 when application of T2T strategy was initiated in our center. Furthermore, a sub-cohort study including T2T and non-T2T period groups was conducted with outcome of cumulative percentage of remission and time to achieve first remission during the first year follow-up.

Results: In total, 1,001 patients with 6,944 clinical visits were included. Over eight-year period, significant improvements were witnessed in disease activity and remission rate, measured by all four indices $(p<0.0001)$. More patients achieved lower disease activity and higher remission rates after T2T adherence in 2011 compared to those in the years of 2009 and $2010(\mathrm{P}<0.0001)$. Moreover, sub-cohort study revealed that more patients $(49.3 \%>73.2 \%$ vs. $19.1 \%>34.5 \%$,
OR=2.4-3.0) achieved remission with a shorter median time compared with the non-T2T period group ( $p<0.0001$ ), particularly in DAS28-CRP ( 21 vs. $>52$ weeks), DAS28-ESR (37 vs. $>52$ weeks).

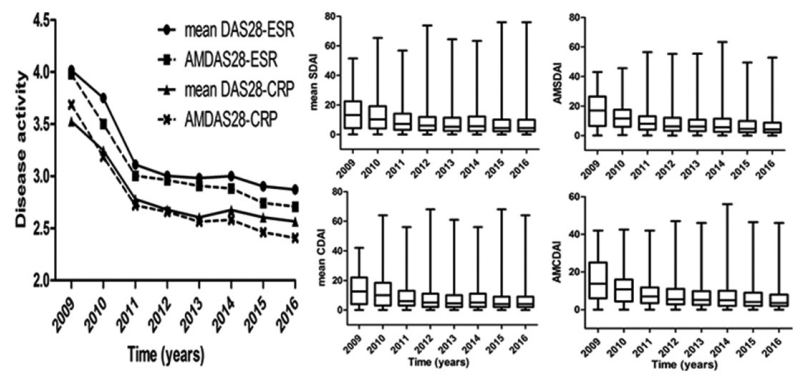

Figure 1. Trends in disease activity scores over 8 years by four indices in mean and adjusted mean (AM) methods. (A) Trends based on DAS28-CRP and DAS28-ESR in two methods (B) Trends based on SDAl and CDAl in two methods

Conclusions: Over past 8 years, the RA activity has substantially decreased and T2T strategy was directly attributable to the favorable changes in clinical practice.

\section{REFERENCES:}

[1] Scott DL, Wolfe F, Huizinga TW. Rheumatoid arthritis. Lancet 2010;376:1094-108.

[2] Grigor C, Capell H, Stirling A, McMahon AD, Lock P, Vallance R, et al. Effect of a treatment strategy of tight control for rheumatoid arthritis (the TICORA study): a single-blind randomized controlled trial. Lancet 2004;364(9430):263-9.

[3] Verstappen SM, Jacobs JW, van der Veen MJ, Heurkens AH, Schenk Y, ter Borg EJ, et al. Intensive treatment with methotrexate in early rheumatoid arthritis: aiming for remission. Computer Assisted Management in Early Rheumatoid Arthritis (CAMERA, an open-label strategy trial). Ann Rheum Dis 2007;66(11):1443-9.

[4] Goekoop-Ruiterman YP, de Vries-Bouwstra JK, Kerstens PJ, et al. DAS driven therapy versus routine care in patients with recent-onset active rheumatoid arthritis. Ann Rheum Dis 2010;69(1):65-9.

Acknowledgements: We would like to thank all the patients, rheumatology nurses and rheumatologists who contributed to our study.

Disclosure of Interest: None declared

DOI: 10.1136/annrheumdis-2018-eular.4050

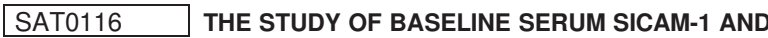 CXCL13 LEVELS IN PREDICTING RESPONSE TO TUMOR NECROSIS FACTOR-A INHIBITOR THERAPY IN PATIENTS WITH RHEUMATOID ARTHRITIS}

X. Ye ${ }^{1}$, J. Zhao ${ }^{1}$, Z. Zhang ${ }^{1} .{ }^{1}$ Rheumatology and Clinical Immunology Department, Peking University First Hospital, Beijing, China

Background: TNF- $\alpha$ inhibitors are not effective for each patient, leading to poor response as well as financial burden. There is an urgent need for biomarkers to assist us in individualized treatment. However, reliable biomarkers that predict therapeutic response to TNF- $\alpha$ inhibitors are still lacking.

Objectives: We aimed to investigate whether baseline serum soluble intercellular adhesion molecule-1 (sICAM-1) and C-X-C motif chemokine ligand 13 (CXCL13) could serve as biomarkers to predict therapeutic response to TNF- $\alpha$ inhibitor therapy in RA patients.

Methods: RA patients were enrolled from the 12-week TNF- $\alpha$ inhibitor clinical trial in our center between October 2014 and October 2017. 20 age- and gendermatched healthy controls were also recruited. Serum samples at baseline and week 12 were collected from RA patients, then serum levels of SICAM-1 and CXCL13 were measured by enzyme-linked immunosorbent assay. Clinical and laboratory data were recorded from baseline to week 12. RA patients were classified into responders and non-responders at week 12 according to EULAR response criteria.

Results: 51 RA patients were enrolled in this study. Serum levels of sICAM-1 and CXCL13 in RA patients were significantly higher than healthy controls $(p<0.001$ and $p<0.001$ respectively). Serum sICAM-1 and CXCL13 levels were higher in seropositive RA patients ( $p=0.012$ and $p=0.005$ respectively). Baseline serum levels of sICAM-1 and CXCL13 were correlated with changes in ESR, DAS28-ESR, DAS28-CRP, SDAI and CDAI. Baseline serum sICAM-1 levels were higher in responders to TNF- $\alpha$ inhibitor therapy at week 12 by EULAR response criteria $(p=0.010)$. However, there was no significant difference in CXCL13 levels. In addition, serum SICAM-1 and CXCL13 levels were decreased after treatment in 
responders $(p<0.001$ and $p<0.001$ respectively), nevertheless, non-responders showed a rising trend $(p=0.086$ and $p=0.051$ respectively). Binary logistic regression model revealed that baseline serum SICAM-1 levels had a positive effect on response to therapy. ROC curve analysis for predictive ability of baseline serum sICAM-1 showed an area under the curve (AUC) of $0.775(p=0.010)$.

Conclusions: Serum sICAM-1 and CXCL13 levels were elevated in RA patients, and they were higher in seropositive patients than in seronegative patients. Elevated baseline serum sICAM-1 levels were associated with favorable response to TNF- $\alpha$ inhibitor therapy. The decrease of serum sICAM-1 levels after treatment in responders was consistent with their therapeutic response. Thus, baseline serum sICAM-1 could be a predictive biomarker for TNF- $\alpha$ inhibitor therapy in RA patients. There was a lack of reliable evidence that baseline serum CXCL13 had predictive ability, possibly due to different mechanisms of action or small sample size.

\section{REFERENCES:}

[1] Zampeli E, Vlachoyiannopoulos PG, Tzioufas AG. Treatment of rheumatoid arthritis: Unraveling the conundrum[J]. J Autoimmun 2015;65:1-18.

[2] Smolen JS, Aletaha D. Rheumatoid arthritis therapy reappraisal: strategies, opportunities and challenges[J]. Nat Rev Rheumatol 2015;11(5):276-89.

[3] Lindstrom TM, Robinson WH. Biomarkers for rheumatoid arthritis: making it personal[J]. Scand J Clin Lab Invest Suppl, 2010;242:79-84.

Acknowledgements: PUCRP 201305

Disclosure of Interest: None declared

DOI: 10.1136/annrheumdis-2018-eular.6015

\section{SATURDAY, 16 JUNE 2018}

\section{Rheumatoid arthritis - comorbidity and clinical aspects}

\section{SAT0117 HIGH URIC ACID AS A RISK FACTOR FOR CARDIOVASCULAR DISEASES IN RHEUMATOID ARTHRITIS PATIENTS}

A. Al-Herz ${ }^{1}$, A. Aldei ${ }^{1}$, K. Saleh ${ }^{2}$, A. Al-Awadhi ${ }^{1}$, W. Al-Kandari ${ }^{2}$, E. Hasan ${ }^{1}$, A. Ghanem ${ }^{3}$, M. Hussain 1 , E. Nahar ${ }^{3}$, F. Abutiban ${ }^{4}$, A. Alenizi ${ }^{4}$, Y. Ali ${ }^{3}$, H. Alhajeri ${ }^{5}$, S. Hayat ${ }^{3}$, A. Khadrawy ${ }^{2}$, A. Fazal ${ }^{2}$, K. Mokaddem ${ }^{1}$, A. Zaman ${ }^{3}$, G. Mazloum ${ }^{5}$ Y. Bartella ${ }^{1}$, S. Hamed ${ }^{1}$, R. Alsouk ${ }^{4}$, A. Al-Saber ${ }^{6} .{ }^{1}$ Rheumatology, Amiri Hospital, Kuwait, ${ }^{2}$ Farwania Hospital, Farwania; ${ }^{3}$ Mubarak Al-Kabeer Hospital, Hawalli; ${ }^{4}$ Jahra Hospital, Jahra; ${ }^{5}$ Mubarak Al-Kabeer Hospital; ${ }^{6}$ Department of Mathematics, Kuwait Technical College, Kuwait, Kuwait

Background: Rheumatoid arthritis (RA) patients have an increased risk of cardiovascular diseases (CVD). It is unclear whether an elevated serum uric acid (UA) further increases that risk.

Objectives: We study CVD and their risk factors in association with UA in RA patients.

Methods: Adult patients who satisfied the ACR classification criteria for RA from The Kuwait Registry for Rheumatic Diseases (KRRD) from four major hospitals were evaluated from February 2013 through May 2017. Patients with recorded UA were identified and CVD and their risk factors were studied in those patients. To optimize classifier number and prediction accuracy, hierarchical cluster analyses for multiple factors were performed, which indicated nine possible independent CVD risk factors. A binary logistic regression was conducted to examine their significant association with $C V D$ and the independence of $U A$ as a risk factor. Results: A total of 564 RA patients with available UA were identified, $353(62.6 \%)$ females. Mean age was $50.8 \pm 11.5$ years and disease duration $10.5 \pm 2.9$ years. Mean UA was $271 \pm 78 \mu \mathrm{mol} / \mathrm{L}$. Of those patients, $31(5.5 \%)$ were reported to have CVD. UA was significantly correlated to the presence of CVD $\left(x^{2}=6.49, p=0.011\right)$. Logistic regression model indicated a $10 \%$ increase of CVD with every $10 \mu \mathrm{mol} / \mathrm{L}$ increase in UA. A correlation matrix between UA and other risk factors showed a significant association between high uric acid and a younger age at RA diagnosis $(r=-0.262)$, hyperlipidemia $(r=0.191)$ and diabetes mellitus $(r=0.244)$.

Conclusions: Our study suggests that UA may be an independent risk factor for CVD and is associated with the presence of other risk factors. UA should be measured and carefully approached in RA patients.

Disclosure of Interest: None declared

DOI: 10.1136/annrheumdis-2018-eular.5694

\section{SAT0118 \\ ASSOCIATION OF RENIN-ANGIOTENSIN SYSTEM IMBALANCE WITH SUBCLINICAL ATHEROSCLEROSIS AND DISEASE ACTIVITY IN RHEUMATOID ARTHRITIS}

N. F. T. Braz ${ }^{1}$, M. R. C. Pinto ${ }^{2}$, É. L. M. Vieira ${ }^{1}$, A. L. Teixeira ${ }^{1}$, A. C. S. Silva ${ }^{1}, \underline{A}$. M. Kakehasi ${ }^{3}{ }^{1}$ Interdisciplinary Laboratory of Medical Investigation, Federal University Of Minas Gerais; ${ }^{2}$ Rheumatology Unit, Clinic Hospital; ${ }^{3}$ Locomotor System Department, Federal University of Minas Gerais, Belo Horizonte, Brazil

Background: Rheumatoid arthritis (RA) is an independent risk factor for cardiovascular disease (CVD). The renin-angiotensin system (RAS) is a hormonal cascade with important role in hydroelectrolytic homeostasis, blood pressure and regulation of cardiovascular remodeling. Angiotensin II (Ang II) acts as a proinflammatory mediator (1).

Objectives: To investigate the association of serum levels of RAS components with the presence of subclinical atherosclerosis using carotid ultrasonography in women with RA.

Methods: Women with RA according to ACR/EULAR 2010 or ACR 1987 criteria and without clinical ischemic CVD were included. Disease activity was assessed using the DAS28. The presence of atherosclerotic plaques and the thickness of the medium-intimal complex (EMI) of the arterial wall in the common carotid artery were evaluated by ultrasonography, Serum levels of angiotensin (Ang) II, Ang-(17), angiotensin converting enzyme (ECA) and ECA II were determined by enzyme immunoassay.

Results: 50 women with RA, mean age 48.2 years $( \pm 7.32)$, mean duration of disease of 15.35 years $( \pm 8.56)$, DAS28 of $4.02( \pm 1.41)$ and CDAI of $14.23( \pm 11.53)$ were included. Seven patients presented altered EMI, eight had atherosclerotic plaque. The prevalence of risk factors for CVD was: $12 \%$ of smoking, $12 \%$ of family history of premature CVD, $46 \%$ of arterial hypertension, $10 \%$ of diabetes, $62 \%$ of dyslipidemia, $94 \%$ of abdominal obesity and $46 \%$ of metabolic syndrome. The control group consisted of 30 healthy women, mean age of 46.3 years $( \pm 7.72)$. RA patients had a higher serum concentration of Ang II $(p<0.01)$, Ang-(1-7) $(p<0.01)$ and ACE $(p<0.01)$ than the control group (table 1). There was a negative correlation between ECA II and EMI ( $\mathrm{p}=0.041$, rho -0.290). EMI correlated positively with age $(p=0.022$, rho 0.324$)$, disease duration $(p=0.012$, rho 0.315$)$ and overall Framingham risk $(p=0.008$, rho 0.368$)$ and Ang II correlated positively with DAS28 $(p=0.034$, rho 0.301) and CDAI ( $p=0.040$, rho 0.291).

Table 1. Comparison between plasma concentrations of SRA biomarkers in patients with rheumatoid arthritis (RA) and the control group (CG).

\begin{tabular}{lcll}
\hline RAS Biomarkers $(\mathrm{pg} / \mathrm{mL})$ & RA $(\boldsymbol{n}=\mathbf{5 0})$ Mean $\pm \mathrm{SD}$ & CG $(\boldsymbol{n}=\mathbf{3 0})$ Mean $\pm \mathrm{SD}$ & $\boldsymbol{p}$ value \\
\hline & & & \\
Ang II & $407.60 \pm 278.68$ & $198.77 \pm 105.48$ & $<0.01^{\mathrm{a}}$ \\
Ang-(1-7) & $162.06 \pm 234.58$ & $36.94 \pm 61.36$ & $<0 . \mathbf{0 1 ^ { \mathrm { a } }}$ \\
ACE & $266.21 \pm 103.64$ & $222.69 \pm 147.61$ & $<0.01^{\mathrm{a}}$ \\
ACE II & $71.86 \pm 27.32$ & $153.57 \pm 225.04$ & $0.17^{\mathrm{a}}$ \\
\hline RA = rheumatoid arthritis; CG= control group; SD= standard deviation; Ang = angiotensin,
\end{tabular}

$\mathrm{ACE}=$ angiotensin converting enzyme. ${ }^{\mathrm{a}}$ Mann-Whitney Test.

Conclusions: Imbalance of RAS components, especially Ang II and ECA II, may be associated to CVD in RA patients. Ultrasonography of the carotid arteries can identify patients that could benefit from ECA blockade.

\section{REFERENCE:}

[1] Chang Y, Wei W. Angiotensin II in inflammation, immunity and rheumatoid arthritis. Clin Exp Immunol 2015;179:137-145. doi: 10.1111/cei.12467

Acknowledgements: National Council for Scientific and Technological Development (CNPq), Foundation for Research Support of Minas Gerais (FAPEMIG) Disclosure of Interest: None declared

DOI: 10.1136/annrheumdis-2018-eular.5624

\section{SAT0119 1 PHYSICAL ACTIVITY IN TUNISIAN ADULTS WITH RHEUMATOID ARTHRITIS}

A. feki ${ }^{1}$, A. fazaa ${ }^{1}$, S. miladi ${ }^{1}$, K. ouenniche ${ }^{1}$, S. kassab ${ }^{1}$, S. chekili ${ }^{1}$, K. ben abdelghani ${ }^{1}$,A. laatar ${ }^{1} .{ }^{1}$ Rheumatology, Mongi Slim Hospital - La Marsa, Tunis, Tunisia

Background: Physical activity (PA) is associated with multiple health-related benefits among the general population and adults with chronic diseases like 